\title{
Nodal surface and persistent spin texture in a Weyl semimetal without mirror symmetry
}

\author{
Wujun Shi ${ }^{1}$ and Gang $\mathrm{Li}^{1,2}$ \\ ${ }^{1}$ School of Physical Science and Technology, ShanghaiTech University, Shanghai 200031, China \\ ${ }^{2}$ ShanghaiTech Laboratory for Topological Physics, ShanghaiTech University, Shanghai 200031, China
}

(Dated: April 16, 2019)

\begin{abstract}
By utilizing symmetry analysis and electronic structure calculations, we investigated the low-temperature orthorhombic phase of $\mathrm{Ag}_{2} \mathrm{Se}$ in $\mathcal{S G}$ 17. In addition to the discovery of a nodal plane at $k_{z}=\pi$ protected by the joint operation of time-reversal $(\mathcal{T})$ and the 2 -fold screw rotation $S_{2 z}$, we found 24 Weyl points mainly residing at the $k_{y}=0$ plane with notable Fermi arc and large quasiparticle interference pattern (QPI). Due to the absence of mirror symmetry, a pair of Weyl points with opposite chirality reside at different binding energies, which makes this system an excellent material candidate for realizing the novel chiral anomaly related phenomenon, such as the quantized circular photogalvanic and the chiral magnetic effects. Furthermore, we also reveal the striking spin textures at $k_{z}=\pi$ plane which demonstrates, in a large region of the surface Brillouin Zone, a direction-selective spin polarization, which has a strong implication to spintronic applications.
\end{abstract}

\section{INTRODUCTION}

Equipped with the predicting power of the topological band theory, the study of topological materials has revolutionized the conventional viewpoint on the state of matter and triggered a great interest in the nontrivial Berry curvature of the Bloch wavefunction $1+12$. Among the many new states of matter, the study of three-dimensional (3D) Weyl semimetal (WSM) has emerged as a new research discipline known as topological semimetal, where the lower-energy excitations around a degenerate Weyl point (WP) between two energy bands are well described as a 3D Weyl fermion. In solids, WPs always appear in pairs of opposite chiralities with quantized Chern number $C^{1}$, acting as the the monopole source and sink of the Berry curvature, which leads to the striking predicitons of the Fermi arc electronic states on the surface $2-9$.

Macroscopically, WPs are known to correlate to many exotic phenomena, such as negative magnetoresistance (also known as chiral anomaly $\sqrt{5 / 13}$, intrinsic spin Hall effect ${ }^{14}$, and anomalous Hall effect ${ }^{15}[17$. Notably, if WPs with opposite chiral charges have different energies the quantized circular photogalvanic effect (CPGE) $\frac{18}{18}$ and the chiral magnetic effect (CME) are also supposed to happen 19, 24, The CME has attracted much theoretical interest because of the existence of a nonzero chiral current induced by a static magnetic field at zero temperature ${ }^{20}$. The CME and CPGE were first proposed by theoretical study and was recently observed in $\mathrm{RhSi}$. Early theoretically proposed material candidates for WSMs have mirror symmetry, which confines the WPs with opposit chiral charges to the same energy. Some recently proposed materials, which do not have mirror symmetry, have the WPs with higher chiral charges $(\geq 1)$, such as the double Weyl fermions in $\mathrm{SrSi}_{2} 26, \mathrm{TlTeO}_{6}$ and $\mathrm{Ag}_{2} \mathrm{Se}$ related families 27 , and the spin-3/2 Rarita-Schwinger-Weyl fermions in $\mathrm{CoSi}$ 281. Therefore, a Weyl semimetal with opposite unit chiral charges being at different energies is highly demanded, which would provide an ideal experimental platform facilitating further examination of those exotic effects in the near future.

In this paper, based on the first-principle calculations and simple symmetry argument, we predict $\mathrm{Ag}_{2} \mathrm{Se}$ at low temperature with space group $(\mathcal{S G}) 17$ as a Weyl semimetal lacking of the protection of mirror symmetry. As a result, the WPs with opposite chirality charge reside at different energies. We found 24 WPs in this material with 20 of WPs locate below Fermi level $\left(E_{F}\right)$. By tuning the energy relative to $E_{F}$, this system can serve as an ideal platform to study the significant CPGE and CME in experiments. The persistent spin current discovered at $k_{z}=\pi$ surface further makes this system a good material candidate for spintronics.

\section{CRYSTAL STRUCTURE AND SYMMETRY.}

At atmospheric pressure, the $\mathrm{Ag}_{2} \mathrm{Se}$ crystallizes in two phases. At high temperature $\mathrm{Ag}_{2} \mathrm{Se}$ crystallizes in the bcc cubic phase $(\operatorname{Im} \overline{3} m)^{\sqrt{32} 33}$. At $406 \mathrm{~K}$ a structure transition to the orthorhombic phase occurs, where $\mathrm{Ag}_{2} \mathrm{Se}$ can have two different structures with $\left.\mathcal{S G} 17\left(P 222_{1}\right)\right)^{33 / 34}$. and $\mathcal{S G} 19$ $\left(P 2_{1} 2_{1} 2_{1}\right)^{33|35| 36}$. The topological properties of $\mathcal{S G} 19$ phase $\mathrm{Ag}_{2} \mathrm{Se}$ has been examined 37. Here, we studied the $\mathcal{S G} 17$ phase whose topological nature, to the best of our knowledge, has not been explored.

Beside identity, $\mathcal{S G} 17$ contains three symmetries: a 2-fold rotation symmetry about $x$ axis, i.e. $C_{2 x}=\left\{2_{100} \mid 0,0,0\right\}$, and two screw rotation symmetries about $y$ and $z$ axis, i.e. $S_{2 y}=\left\{2_{010} \mid 0,0, \frac{1}{2}\right\}$ and $S_{2 z}=\left\{2_{001} \mid 0,0, \frac{1}{2}\right\}$. Their operations are expressed as: $C_{2 x}:(x, y, z) \rightarrow(x,-y,-z) \otimes\left(-i \sigma_{x}\right)$, $S_{2 y}:(x, y, z) \rightarrow(-x, y,-z+c / 2) \otimes\left(-i \sigma_{y}\right), S_{2 z}:(x, y, z) \rightarrow$ $(-x,-y, z+c / 2) \otimes\left(-i \sigma_{z}\right)$, where the spin manipulation is indicated by the corresponding Pauli matrices. Due to the absence of inversion symmetry and the Kramer degeneracy, at generic momentum every Bloch band is non-degenerate. However, higher band degeneracy can still be expected in this $\mathcal{S} G$ : (1) each band at the eight time-reversal invariant momenta (TRIM) are doubly degenerate protected by the time-reversal symmetry $\mathcal{T}$, i.e. $|\Psi\rangle$ and $\mathcal{T}|\Psi\rangle$ are orthogonal and energetically degenerate at TRIM. (2) Screw symmetry $S_{2 z}$ protects crossings between two bands carrying different $S_{2 z}$ eigenvalues. It is easy to understand that, after applying $S_{2 z}$ twice, every atom moves along $z$ axis with one lattice spacing, resulting in an additional phase factor in the Bloch wavefunction, i.e. $S_{2 z}^{2}|\Psi(k)\rangle=-e^{i k_{z}}|\Psi(k)\rangle$ with the minus 


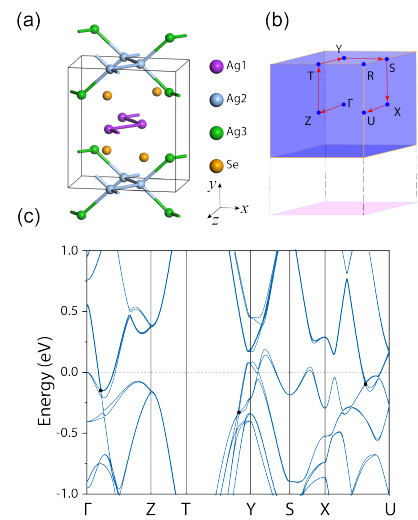

(d)
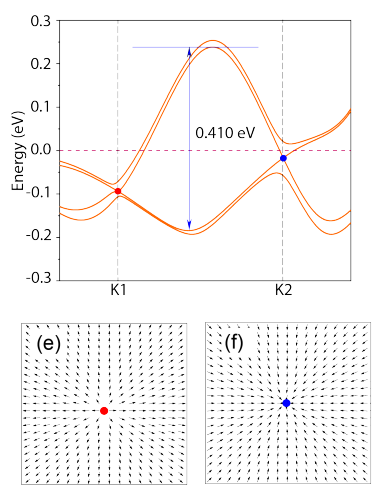

FIG. 1. The lattice structure of the $\mathrm{Ag}_{2} \mathrm{Se}$ (a) and its BZ (b) with the $(010)$ projection. The bulk band structure with SOC is shown in (c) along high-symmetry path indicated in (b). (d) illustrates a pair of WPs K1 (0.500 $0.000-0.315)$, K2 (0.195 $0.000-0.153)$ in the unit $\left(\frac{2 \pi}{a}, \frac{2 \pi}{b}, \frac{2 \pi}{c}\right)$ and the two associated bands inverted in between with the inversion energy $0.41 \mathrm{eV}$. (e) and (f) demonstrate the berry curvature associated with these two WPS. In (c), the black dots indicate the band cross, and the dashed line indicates the Fermi level.

sign from the spin operation. As $S_{2 z}$ commutes with the Hamiltonian, each Bloch state can be labelled by the eigenvalue of $S_{2 z}$ as well. Although $k_{z}= \pm \pi$ are equivalent Brillouin Zone (BZ) boundaries, the eigenvalue of $S_{2 z}$ takes different values, i.e. $\pm i e^{i k_{z} / 2}= \pm 1$ at $k_{z}=-\pi$ and $\pm i e^{i k_{z} / 2}=\mp 1$ at $k_{z}=\pi$. Thus, to satisfy the periodic boundary condition of the BZ the bands with opposite $S_{2 z}$ eigenvalues shall always appear in pairs ${ }^{38}$ and cross at somewhere along the high symmetry lines protected by $S_{2 z}$. There are four k-paths of this type protected with band crossings, i.e. $\left(0 / \pi, 0 / \pi, k_{z}\right)$.

(3) At $k_{z}=\pi$ plane every band are doubly degenerate due to the protection of the joint protection of $\mathcal{T}$ and $S_{2 z}$, i.e. there exists a nodal plane in $\mathcal{S G} 17$ as shown below. After defining $\Theta_{z}=\mathcal{T} S_{2 z}$, one can easily prove that the momentum at $k_{z}=0 / \pi$ planes are invariant under $\Theta_{z}$, $\left(k_{x}, k_{y}, 0 / \pi\right) \stackrel{S_{2 z}}{\longrightarrow}\left(-k_{x},-k_{y}, 0 / \pi\right) \stackrel{\mathcal{T}}{\rightarrow}\left(k_{x}, k_{y}, 0 / \pi\right)$. While, in real-space after applying $\Theta_{z}$ twice the $x$ and $y$ coordinates are kept invariant, but $z$ coordinate will be shifted by one lattice spacing $c,(x, y, z) \stackrel{S_{2 z}}{\longrightarrow}(-x,-y, z+c / 2) \stackrel{\mathcal{T}}{\rightarrow}(-x,-y, z+c / 2)$. As a result, for each Bloch band $\Theta_{z}^{2}|\Psi(k)\rangle=e^{i k_{z}}|\Psi(k)\rangle$. At $k_{z}=\pi$ plane, $\Theta_{z}^{2}=-1$ acting as a time-reversal operator which allows a double band degeneracy everywhere in this plane. The other screw rotation, i.e. $S_{2 y}$, however does not bring in any additional band degeneracy, as applying $\Theta_{y}=\mathcal{T} S_{2 y}$ twice will not result in additional lattice translation so that $\Theta_{y}^{2}=1$.

(4) What is more striking about $\mathcal{S G} 17$ is the protection of nonequilibrium spin polarization along the boundary of $k_{z}=\pi$ surface, which strongly connects to the nodal nature of this plane, resulting in a persistent spin texture allowed only in selective directions in momentum space. As $\Theta_{z}|\Psi\rangle$ is energetically degenerate with $|\Psi\rangle$, it is easy to verify $s_{z}=\left\langle\Psi\left|\Theta_{z}^{\dagger} \hat{\sigma}_{z} \Theta_{z}\right| \Psi\right\rangle=-\left\langle\Psi\left|\hat{\sigma}_{z}\right| \Psi\right\rangle$ due to the fact that $\hat{\sigma}_{z}$ anticommutes with $\mathcal{T}$ and commutes with $S_{2 z}$. Adding up the contributions from the two degenerate states yields a vanishing spin polarization along $z$ direction. However, the other two spin components survive as $\left\{S_{2 z}, \hat{\sigma}_{x / y}\right\}=0$. We note that this strongly contrasts to the spin textures in centrosymmetric systems, where the Kramer's degeneracy enforces the doubly degenerate bands to have perfectly opposite spin textures. Therefore a cancellation of spin polarization happens everywhere in the BZ. Here the $\Theta_{z}$ protected nodal plane promotes the cancellation only for the $\left\langle S_{z}\right\rangle$ component, leaving the spin momentum fully planar.

\section{BULK ELECTRONIC STRUCTURE}

For systems in this $\mathcal{S G}$ the more interesting thing is the presence of WPs at generic momentum due to the broken of inversion symmetry, which cannot be obtained from the above symmetry analysis. To this end, we calculated the electronic band structure and search the possible WPs in the first BZ by using the density functional theory (DFT) as implemented in the Vienna ab-initio Simulation Package (VASP) ${ }^{39}$ with projector augmented wave method $40 \mid 41$. The electron exchange-correlation potential is described by the generalized gradient approximation of Perdew-Burke-Ernzerhof (GGA-PBE) scheme ${ }^{42}$. The kinetic energy cutoff of the plane wave basis is set to $300 \mathrm{eV}$ as default. A $\Gamma$-centered $6 \times 6 \times 10 k$-mesh is used to sample the BZ. To calculate the surface states, the surface Green's function method was employed with the tight-binding Hamiltonian constructed by the maximally localized Wannier orbitals ${ }^{43 / 44}$, where the Se $s, p$; and Ag $s, p$, and $d$ orbitals were considered. The experiment lattice parameters $(a=7.050 \AA, b=7.850 \AA$, and $c=4.330 \AA$ ) were employed in the calculations with the crystal structure shown in Fig. 11(a). The BZ and the corresponding (010) surface BZ are displayed in Fig. 1.b). The electronic structure calculated with spin-orbit coupling (SOC) along the high symmetry path is shown in Fig.1 (c).

Bloch states along $\Gamma-\mathrm{Z}, \mathrm{T}-\mathrm{Y}$ and $\mathrm{X}-\mathrm{U}$ are the eigenstates of $S_{2 z}$ screw symmetry, therefore there will be $S_{2 z}$ enforced band crossings at these three lines as explained before. Along each line we marked one such crossing point by black dot and we note that each band will at least have one crossing point with another band carrying different $S_{2 z}$ eigenvalues. However, their locations in momentum-energy space are unpredictable by symmetry and is material-dependent. In addition to the $S_{2 z}$ symmetry-enforced band crossing at high-symmetry lines confirmed by our DFT calculations, the $\Theta_{z}=\mathcal{T} S_{2 z}$ protected nodal plane $\left(k_{z}=\pi\right)$ was also verified numerically, where every band is confirmed to be doubly degenerate.

In Fig. 2(a) we show the bottom conduction band and the top valence band at $k_{z}=\pi$ plane, where each band is doubly degenerate and spin momentum contains only in-plane components, i.e. $\left\langle S_{x}\right\rangle$ and $\left\langle S_{y}\right\rangle$. For a better visualization, the spin texture for the valence band is projected to the $k_{x}-k_{z}$ plane. We first note that, along $k_{y}=0, \pi$ lines, only the $\left\langle S_{y}\right\rangle$ component is nonzero. This is due to the additional constraint from $S_{2 y}$ which keeps the momentum invariant along these lines and it commute with the Hamiltonian. Each eigenstate 

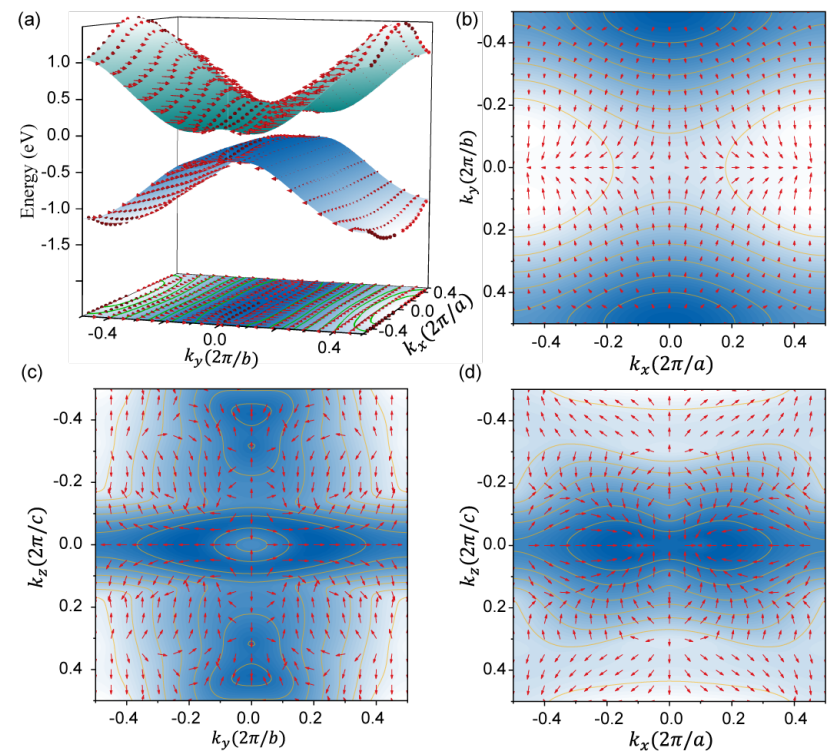

FIG. 2. Spin texture of the top valence band at (a, b) $k_{z}=\pi$; (c) $k_{x}=\pi$ and (d) $k_{y}=\pi$ planes. In addition, in (a) the spin texture of the bottom conduction band is also shown and further overlaid on the band surface.

of the Hamiltonian, thus, will carry a definite eigenvalue of $S_{2 y}$ as well. From $S_{2 y}^{2}=-1$, the eigenvalue of $S_{2 y}$ can be either $i$ or $-i$, which classifies the Bloch states into two groups. For a given state $\left|\Psi^{(+)}\right\rangle$, for example with $S_{2 y}$ eigenvalue $i$, the anti-commutation relation $\left\{\sigma_{y}, \sigma_{x}\right\}=0$ results in $\left\langle S_{x}\right\rangle \sim\left\langle\Psi^{(+)}\left|\sigma_{x}\right| \Psi^{(+)}\right\rangle=\left\langle\Psi^{(+)}\left|S_{2 y}^{\dagger} \sigma_{x} S_{2 y}\right| \Psi^{(+)}\right\rangle=$ $-\left\langle\Psi^{(+)}\left|\sigma_{x}\right| \Psi^{(+)}\right\rangle=0$. As a consequence, along $k_{y}=0 / \pi$ lines at $k_{z}=\pi$ plane the only non-zero component of the spin is $\left\langle S_{y}\right\rangle$, which forces the spin transports only along $k_{y}$ direction. As displayed in Fig. 2(b), the spin texture along these lines are perfectly aligning to $k_{y}$ direction only. The transport of spin current would then be protected by the symmetry against impurities and defects scattering. More interestingly, we find that, in $\mathrm{Ag}_{2} \mathrm{Se}$, the persistent of spin texture extends to a large regime around these lines, making the majority of spin texture at $k_{z}=\pi$ plane orientated to $k_{y}$. The persistent spin texture has been predicted to support long spin lifetime and create non-equilibrium spin polarization ${ }^{45}$ which is crucial for spintronic applications 46 . In the other two planes, i.e. $k_{x}=\pi$ in Fig. 2(c) and $k_{y}=\pi$ in Fig. 2(d), the spin textures are three dimensional and are much more random than the $k_{z}=\pi$ plane.

\section{WEYL POINTS, SURFACE STATES AND QPI}

Furthermore, we found 24 WPs around the Fermi level, see Table I for their positions in momentum-energy space. These WPs are symmetry related. Given a WP at an arbitrary $k$ point $\left(k_{x}, k_{y}, k_{z}\right), e . g ., C_{2 x}$ rotation symmetry and $\mathcal{T}$ guarantee that there will be three more WPs locating at $\left(k_{x},-k_{y},-k_{z}\right)$, $\left(-k_{x},-k_{y},-k_{z}\right)$, and $\left(-k_{x}, k_{y}, k_{z}\right)$. As neither $C_{2}$ and $\mathcal{T}$ changes Chern number, all these symmetry-connected WPs
TABLE I. The six groups of $\mathcal{S G} 17 \mathrm{Ag}_{2} \mathrm{Se}$ WPs. The WP position (in reduced coordinates $x, y, z$ ), Chern number, and the energy relative to the Fermi energy $\left(E_{F}\right)$. For each group, there are four symmetry-related WPs.

\begin{tabular}{cccc}
\hline \hline WPs & $\begin{array}{c}\text { Coordinates } \\
\left(\frac{2 \pi}{a}, \frac{2 \pi}{a}, \frac{2 \pi}{c}\right)\end{array}$ & $\begin{array}{c}E-E_{F} \\
(\mathrm{eV})\end{array}$ & $\begin{array}{c}\text { Chern } \\
\text { number }\end{array}$ \\
\hline$W_{1}^{+}(0.082,0.000,0.139)$ & -0.191 & +1 \\
$W_{2}^{+}$ & $(0.195,0.000,0.153)$ & -0.017 & +1 \\
$W_{3}^{+}$ & $(0.462,0.000,0.416)$ & -0.043 & +1 \\
$W_{4}^{-}$ & $(0.026,0.000,0.118)$ & -0.199 & -1 \\
$W_{5}^{-}$ & $(0.118,0.500,0.034)$ & 0.200 & -1 \\
$W_{6}^{-}$ & $(0.500,0.000,0.315)$ & -0.094 & -1 \\
\hline \hline
\end{tabular}

have the same chirality. Therefore, the 24 WPs can be devided into six groups. Within each group, the WPs are related by $\mathcal{T}$ and $C_{2}$. However, WPs from different groups have different energies due to the broken of mirror symmetry. Thus, a net chirality charge can be achieved by tuning the Fermi level in this system, which has a strong implication to the surface states and the realization of CME and CPGE at low temperature. To see clearly the energy difference between a pair of WPs, we show in Fig. 1(d) the bands connecting a chosen pair of WPs. The location of two WPs $\left(K_{1}\right.$ and $\left.K_{2}\right)$ are denoted in this figure by the red and blue dots. The energy separation of the two WPs is as large as $70 \mathrm{meV}$. We also notice that the two bands forming this pair of WPs exchange orders between $K_{1}$ and $K_{2}$ resulting in a band inversion, whose strength is characterized by an energy gap of $0.410 \mathrm{eV}$. In Fig. 1 (e) and (f) the Berry curvature around this pair of WPs demonstrate clear opposite chirality.

The characteristic feature of Weyl systems is the charge transport between the Weyl points through the Fermi arc. In Fig. 3 the states at the Fermi level are displayed for (010) surface. We find four arcs as highlighted inside the dashed line box where their spin textures are also displayed. As the WPs reside at different energies, it is impossible to get a pair of WPs on the same energy cut. Instead, one can inspect the relation of the Fermi arcs with the Chern number by introducing momentum cut. In Fig. 3. b) we show the locations of the 24 WPs in the surface BZ. We use square and circle to denote the positive and negative chirality. The different colors represent the different groups. We note that some WPs are not visible in this plot as they stay vertically below/above some of these WPs in energy. As shown in Fig. 3. b), we introduce four momentum cuts along $k_{x}$ axis. Each momentum cut separates the BZ into two parts, each of which encloses different number of WPs yielding a net Chern number. Consequently, there will be surface states passing through this momentum cut if the enclosed Chern number is nonzero. The number of the topological surface states must be same as the enclosed Chern number. The calculated results are displayed in Fig. 3. (c-f). For cut $\# 1$, the enclosed Chern number is +2 , therefore, will be 2 surface states. For cut \#2, the there are four topological surface states, it is consistent with the enclosed Chern number +4 , while for cut \#3, the Chern number decreases to +2 because of two more -2 Chern number WPs are enclosed, there are 

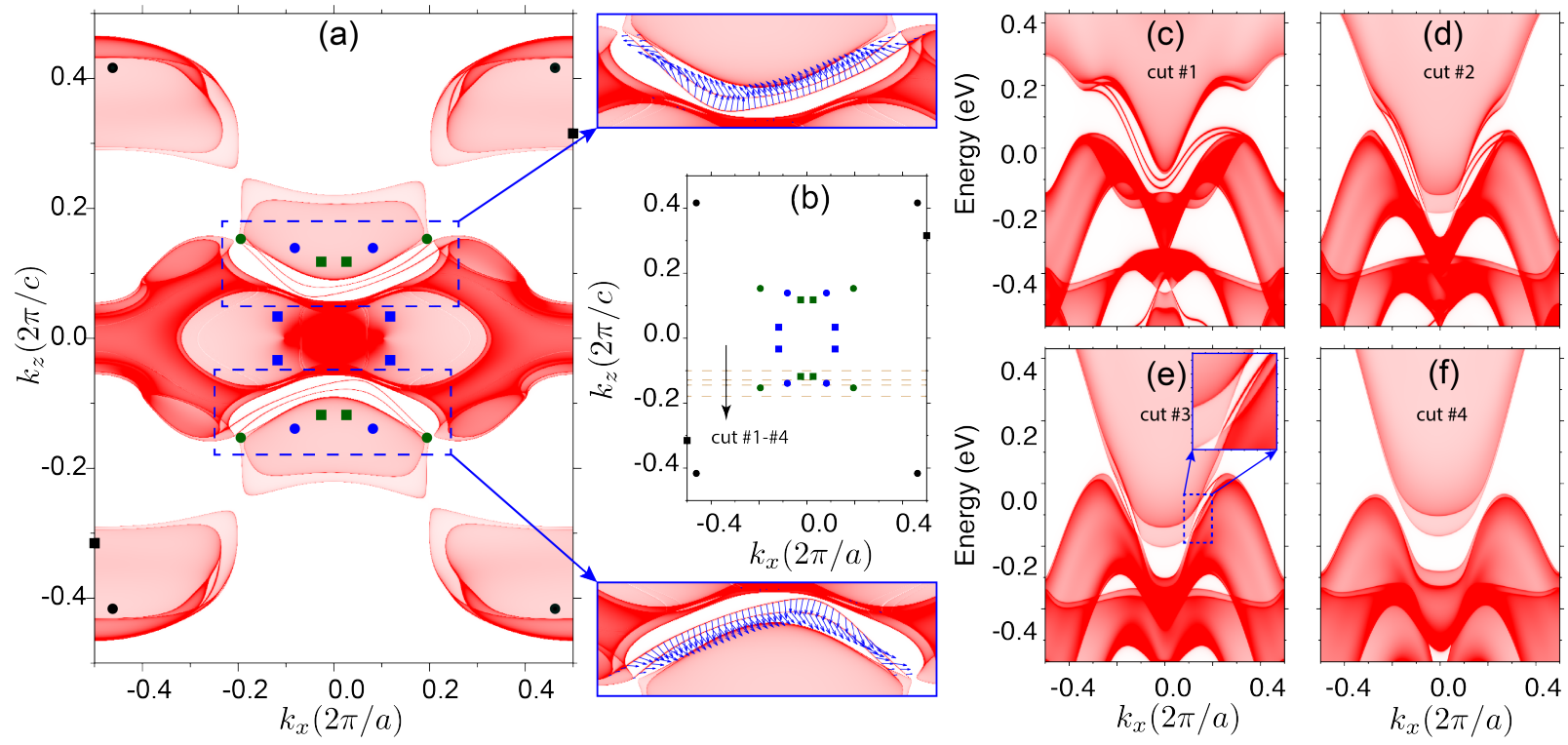

FIG. 3. (a) The calculated surface states at the Fermi level and the (010) surface with the Fermi arcs and their spin texture highlighted in the dashed line box. The location of the 24 WPs are shown in (b) with four momentum cuts, each of which separats the WPs into two parts with different net Chern numbers. Along the four momentum cuts, the corresponding electronic states are shown in (c-f) with different number of surface states highly consistent with the prediction of the enclosed Chern number by the momentum cut. In (a) and (b), the filled squares and circles indicate the WPs with opposite chiral charges and different color indicate the different groups, and the black solid arrows indicate two WPs projected together with the net chiral charges of $|C|=2$, while other WPs without arrows indicates the net chiral charges of $|C|=1$.

two topological surface states. For cut \#4, the enclosed Chern number is zero. Consequently, there is no topological surface state at all passing through this momentum cut.
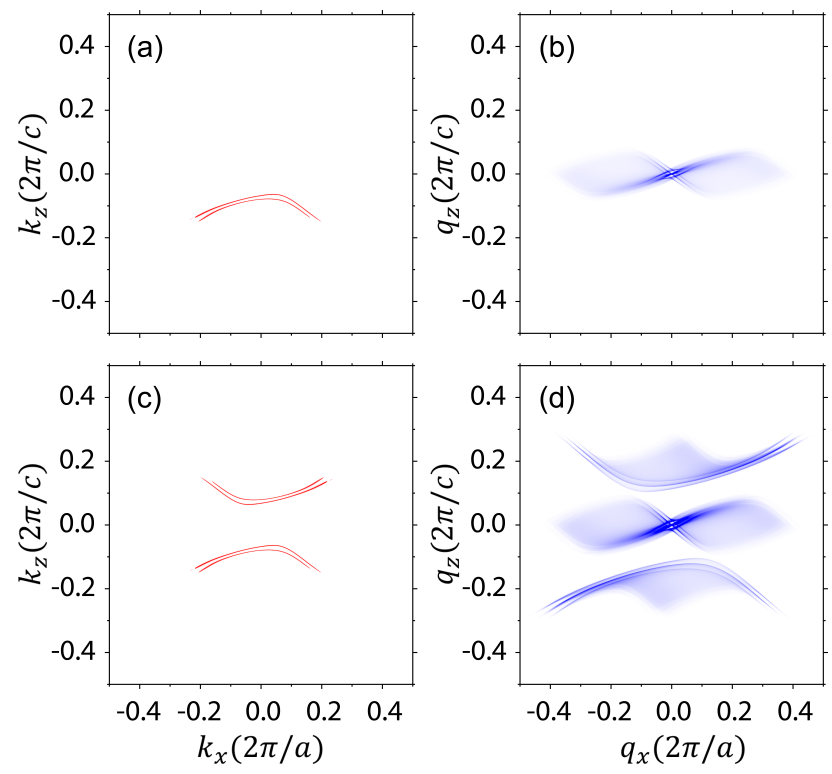

FIG. 4. Quasiparticle interference pattern induced by the Fermi arc states shown in Fig. 3 a). One branch of the fermi arc (a) leads to the scattering among the states within it, whose QPI (b) is distributed only around $\Gamma$ point. The scattering between the two branches of the Fermi arcs shown in (c) leads to the additional QPI mode locating at higher $q_{z}$ value (d).
Through both energy- and momentum-cut we have verified that the discovered 24 WPs are indeed topological and $\mathrm{Ag}_{2} \mathrm{Se}$ in $\mathcal{S G} 17$ is a Weyl semimetal holding great potential for CME and CPGE. To help experimental characterization and verification of this topologically nontrivial system, we further calculate the quasiparticle interference (QPI) pattern which can be directly probed by scanning tunneling spectroscopy (STM). QPI measures the scattering pattern between the initial surface states at $k_{i}$, and the final states at $k_{f}$ at a fixed energy, which is theoretically represented by the joint density of states (JDOS) ${ }^{47}$ $\operatorname{JDOS}(q, E)=\int \frac{d^{3} k}{(2 \pi)^{3}} \sum_{\sigma} A_{\sigma \sigma}(k, E) \sum_{\sigma^{\prime}} A_{\sigma^{\prime} \sigma^{\prime}}(k+q, E)$, where $A_{\sigma \sigma}(k, E) \sum_{\sigma^{\prime}}$ is the Fourier-transformed density of states with spin $\sigma\left(\sigma^{\prime}\right)$ at $k$ point and energy $E$, which can be obtain from the imaginary part of the surface Green's function. To trace the scattering pattern resulted solely by the topological nature of this system, we only consider the QPI between the surface Fermi arc shown in Fig. 3 (a). For a better understanding, we dissemble the Fermi arc shown in Fig. 3 and calculate the JDOS associated with them. With only one branch of the Fermi arcs, scattering is allowed only between the states within this arc. As shown in Fig. 4(b) the Fermi arc in Fig. 4 results in a bow tie shape QPI distributing around the BZ center. With the inclusion of the other branch shown in Fig. 4 (c), the scattering between the two Fermi arcs is now allowed, which additionally leads to the QPI patten locating above/below the bow tie QPI. The entire QPI spans over a large territory of the surface $\mathrm{BZ}$, which provides a great opportunity for STM to detect it. We hope this would stimulate interest for relevant experimental techniques. 


\section{CONCLUSION}

We have studied the $\mathcal{S G} 17$ whose symmetry operation is simple but astonishingly rich phases can be derived which have strong implication to many exciting phenomena. We found a symmetry protected nodal plane in this noncentrosymmetric system protected by the joint operation of time-reversal $\mathcal{T}$ and screw rotation $S_{2 z}$ symmetries. Along the edge of the nodal plane, a direction selective spin polarization is achieved. Unlike the quantum spin Hall system, the polarization of the spin current does not require the presence of perfectly long edge which is technically hard to achieve. The direction selective spin polarization is a bulk quantity determined completely by the symmetry. The presence of persistent spin texture in momentum space may utilize potential applications in spintronics. By using the first-principle calculations, we also found 24 WPs locating at generic momentum with notable Ferm arcs and large QPI. The absence of mirror symmetry makes this system also a perfect material platform for quantized circular photogalvanic and the chiral magnetic effect.

\section{ACKNOWLEDGMENTS}

W.S. would like to thank Max Planck Institute for Chemical Physics of Solids at Dresden for the hospitality where part of this work was done. W.S. wants to acknowledge Yan Sun and Claudia Felser for the fruitful collaborations in other projects. G.L. wants to thank Shilei Zhang for helpful discussions on the momentum-space Skyrmion texture. G.L. acknowledges the starting grant of ShanghaiTech University and the Program for Professor of Special Appointment (Shanghai Eastern Scholar). Calculations were partly carried out at the HPC Platform of ShanghaiTech University Library and Information Services, as well as School of Physical Science and Technology.
1 Xian Gang Wan, Ari M Turner, Ashvin Vishwanath, and Sergey Y Savrasov, "Topological semimetal and Fermi-arc surface states in the electronic structure of pyrochlore iridates," Phys. Rev. B 83, 205101 (2011).

2 Hongming Weng, Chen Fang, Zhong Fang, B. Andrei Bernevig, and Xi Dai, "Weyl Semimetal Phase in Noncentrosymmetric Transition-Metal Monophosphides," Phys. Rev. X 5, 011029 (2015).

3 Shin-Ming Huang, Su-Yang Xu, Ilya Belopolski, Chi-Cheng Lee, Guoqing Chang, BaoKai Wang, Nasser Alidoust, Guang Bian, Madhab Neupane, Chenglong Zhang, Shuang Jia, Arun Bansil, Hsin Lin, and M Z Hasan, "A Weyl Fermion semimetal with surface Fermi arcs in the transition metal monopnictide TaAs class," Nat. Commun. 6, 8373 (2015).

4 B Q Lv, H M Weng, B B Fu, X P Wang, H Miao, J Ma, P Richard, X C Huang, L X Zhao, G F Chen, Z. Fang, X. Dai, T Qian, and H Ding, "Experimental Discovery of Weyl Semimetal TaAs," Phys. Rev. X 5, 031013 (2015).

5 Su-Yang Xu, Ilya Belopolski, Nasser Alidoust, Madhab Neupane, Guang Bian, Chenglong Zhang, Raman Sankar, Guoqing Chang, Yuan Zhujun, Chi-Cheng Lee, Huang Shin-Ming, Hao Zheng, Jie Ma, Daniel S. Sanchez, BaoKai Wang, Arun Bansil, Fangcheng Chou, Pavel P. Shibayev, Hsin Lin, Shuang Jia, and M. Zahid Hasan, "Discovery of a Weyl fermion semimetal and topological Fermi arcs," Science 349, 613 (2015).

6 Zhijun Wang, Dominik Gresch, Alexey A Soluyanov, Weiwei Xie, S Kushwaha, Xi Dai, Matthias Troyer, Robert J Cava, and B. Andrei Bernevig, "MoTe2: A Type-II Weyl Topological Metal," Phys. Rev. Lett. 117, 056805 (2016).

7 Y. Sun, S C Wu, M N Ali, C. Felser, and B Yan, "Prediction of Weyl semimetal in orthorhombic MoTe 2," Phys. Rev. B 92, 161107(R) (2015).

8 Alexey A Soluyanov, Dominik Gresch, Zhijun Wang, Quansheng Wu, Matthias Troyer, Xi Dai, and B. Andrei Bernevig, "Type-II Weyl semimetals," Nature 527, 495-498 (2015).

9 Juan Jiang, ZK Liu, Y Sun, HF Yang, CR Rajamathi, YP Qi, LX Yang, C Chen, H Peng, CC Hwang, et al., "Signature of type-ii weyl semimetal phase in mote2," Nat. Commun. 8, 13973 (2017).
10 Oskar Vafek and Ashvin Vishwanath, "Dirac Fermions in Solids: From High-Tc Cuprates and Graphene to Topological Insulators and Weyl Semimetals," Annu. Rev. Condens. Matter Phys. 5, 83-112 (2014).

11 Zhijun Wang Wang, M. G. Vergniory, S. Kushwaha, Max Hirschberger, and E. V. Chulkov, "Time-reversal-breaking weyl fermions in magnetic heusler alloys," Phys. Rew. Lett. 117, 236401 (2016).

12 NP Armitage, EJ Mele, and Ashvin Vishwanath, "Weyl and dirac semimetals in three dimensional solids," arXiv preprint arXiv:1705.01111 (2017).

13 Su-Yang Xu, Nasser Alidoust, Ilya Belopolski, Zhujun Yuan, Guang Bian, Tay-Rong Chang, Hao Zheng, Vladimir N. Strocov, Daniel S. Sanchez, Guoqing Chang, Chenglong Zhang, Daixiang Mou, Yun Wu, Lunan Huang, Chi-Cheng Lee, Shin-Ming Huang, BaoKai Wang, Arun Bansil, Horng-Tay Jeng, Titus Neupert, Adam Kaminski, Hsin Lin, Shuang Jia Jia, and M. Zahid Hasan, "Discovery of a Weyl fermion state with Fermi arcs in niobium arsenide," Nat. Phys. 11, 748 (2015).

14 Yan Sun, Yang Zhang, Claudia Felser, and Binghai Yan, "Strong intrinsic spin Hall effect in the TaAs family of Weyl semimetals," Phys. Rev. Lett. 117, 146403 (2016).

15 A A Burkov and Leon Balents, "Weyl Semimetal in a Topological Insulator Multilayer,” Phys. Rev. Lett. 107, 127205 (2011).

16 Gang Xu, Hongming Weng, Zhijun Wang, Xi Dai, and Zhong Fang, "Chern Semimetal and the Quantized Anomalous Hall Effect in $\mathrm{HgCr}_{2} \mathrm{Se}_{4}$," Phys. Rev. Lett. 107, 186806 (2011).

17 Yang Zhang, Yan Sun, Hao Yang, Jakub ?elezny, Stuart P. P. Parkin, Claudia Felser, and Binghai Yan, "Strong anisotropic anomalous hall effect and spin hall effect in the chiral antiferromagnetic compounds mn $3 \mathrm{x}(\mathrm{x}=\mathrm{ge}, \mathrm{sn}, \mathrm{ga}$, ir, rh, and pt)," Phys. Rew. B 95, 075128 (2017).

18 Fernando de Juan, Adolfo G Grushin, Takahiro Morimoto, and Joel E Moore, "Quantized circular photogalvanic effect in weyl semimetals,” Nat. Commun. 8, 15995 (2017).

19 Kenji Fukushima, Dmitri E. Kharzeev, and Harmen J. Warringa, "Chiral magnetic effect," Phys. Rev. D 78, 074033 (2008)

20 Gök çe Başar, Dmitri E. Kharzeev, and Ho-Ung Yee, "Triangle anomaly in weyl semimetals," Phys. Rev. B 89, 035142 (2014) 
21 Pallab Goswami, Girish Sharma, and Sumanta Tewari, "Optical activity as a test for dynamic chiral magnetic effect of weyl semimetals," Phys. Rev. B 92, 161110 (2015)

22 Ming-Che Chang and Min-Fong Yang, "Chiral magnetic effect in a two-band lattice model of weyl semimetal," Phys. Rev. B 91, 115203 (2015)

23 Y. Chen, Si Wu, and A. A. Burkov, "Axion response in weyl semimetals," Phys. Rev. B 88, 125105 (2013)

24 M. M. Vazifeh and M. Franz, "Electromagnetic response of weyl semimetals," Phys. Rev. Lett. 111, 027201 (2013)

25 Dylan Rees, Kaustuv Manna, Baozhu Lu, Takahiro Morimoto, Horst Borrmann, Claudia Felser, J. E. Moore, Darius H. Torchinsky, and J. Orenstein, "Quantized Photocurrents in the Chiral Multifold Fermion System RhSi," arXiv e-prints , arXiv:1902.03230 (2019), arXiv:1902.03230 [cond-mat.mes-hall]

26 Shin-Ming Huang, Su-Yang Xu, Ilya Belopolski, Chi-Cheng Lee, Guoqing Chang, Tay-Rong Chang, BaoKai Wang, Nasser Alidoust, Guang Bian, Madhab Neupane, Daniel Sanchez, Hao Zheng, Horng-Tay Jeng, Arun Bansil, Titus Neupert, Hsin Lin, and M. Zahid Hasan, "New type of weyl semimetal with quadratic double weyl fermions," Proc. Natl Acad. Sci. USA 113, 1180-1185 (2016)

21 Guoqing Chang, Bahadur Singh, Su-Yang Xu, Guang Bian, Shin-Ming Huang, Chuang-Han Hsu, Ilya Belopolski, Nasser Alidoust, Daniel S Sanchez, Hao Zheng, et al., "Theoretical prediction of magnetic and noncentrosymmetric weyl fermion semimetal states in the $r-a l-x$ family of compounds $(r=$ rare earth, $\mathrm{al}=$ aluminium, $\mathrm{x}=\mathrm{si}$, ge)," arXiv preprint arXiv:1604.02124 (2016).

28 Barry Bradlyn, Jennifer Cano, Zhijun Wang, M. G. Vergniory, C. Felser, R. J. Cava, and B. Andrei Bernevig, "Beyond dirac and weyl fermions: Unconventional quasiparticles in conventional crystals," Science 353 (2016), 10.1126/science.aaf5037

29 Peizhe Tang, Quan Zhou, and Shou-Cheng Zhang, "Multiple types of topological fermions in transition metal silicides," Phys. Rev. Lett. 119, 206402 (2017)

30 DA Pshenay-Severin, Yu V Ivanov, AA Burkov, and AT Burkov, "Band structure and unconventional electronic topology of cosi," arXiv preprint arXiv:1710.07359 (2017).

31 Guoqing Chang, Su-Yang Xu, Benjamin J Wieder, Daniel S Sanchez, Shin-Ming Huang, Ilya Belopolski, Tay-Rong Chang, Songtian Zhang, Arun Bansil, Hsin Lin, et al., "Large fermi arcs in unconventional weyl semimetal rhsi," arXiv preprint arXiv:1706.04600 (2017).

32 M Oliveria, RK McMullan, and BJ Wuensch, "Single crystal neutron diffraction analysis of the cation distribution in the high-temperature phases $\alpha$-cu2-xs, $\alpha$-cu2-xse, and $\alpha$-ag2se," Solid State Ionics 28, 1332-1337 (1988).
33 Yu G Asadov, Yu I Aliyev, and AG Babaev, "Polymorphic transformations in cu 2 se, ag 2 se, agcuse and the role of partial cation-cation and anion-anion replacement in stabilizing their modifications," Physics of Particles and Nuclei 46, 452-474 (2015).

34 Y Baer, G Busch, C Fröhlich, and E Steigmeier, "Wärmeleitfähigkeit, elektrische leitfähigkeit, hall-effekt, thermospannung und spezifische wärme von ag2se," Zeitschrift für Naturforschung A 17, 886-889 (1962).

35 GA Wiegers, "The crystal structure of the low-temperature form of silver selenide," American Mineralogist: Journal of Earth and Planetary Materials 56, 1882-1888 (1971).

36 Jaemin Yu and Hoseop Yun, "Reinvestigation of the low-temperature form of $\mathrm{Ag}_{2} \mathrm{Se}$ (naumannite) based on single-crystal data," Acta Crystallographica Section E 67, i45 (2011)

$3 /$ Jihwan Kim, Ahreum Hwang, Sang-Hoon Lee, Seung-Hoon Jhi, Sunghun Lee, Yun Chang Park, Si-in Kim, Hong-Seok Kim, Yong-Joo Doh, Jinhee Kim, and Bongsoo Kim, "Quantum electronic transport of topological surface states in $\beta$-ag2se nanowire," ACS Nano 10, 3936-3943 (2016)

38 Steve M. Young and Charles L. Kane, "Dirac semimetals in two dimensions," Phys. Rev. Lett. 115, 126803 (2015)

39 G. Kresse and J. Hafner, "Ab initio molecular dynamics for liquid metals," Phys. Rev. B 47, 558-561 (1993)

40 P. E. Blöchl, "Projector augmented-wave method," Phys. Rev. B 50, 17953-17979 (1994)

41 G. Kresse and D. Joubert, "From ultrasoft pseudopotentials to the projector augmented-wave method," Phys. Rev. B 59, 1758-1775 (1999)

42 John P. Perdew, Kieron Burke, and Matthias Ernzerhof, "Generalized gradient approximation made simple," Phys. Rev. Lett. 77, 3865-3868 (1996)

43 Arash A Mostofi, Jonathan R Yates, Young-Su Lee, Ivo Souza, David Vanderbilt, and Nicola Marzari, "wannier90: A tool for obtaining maximally-localised wannier functions," Computer physics communications 178, 685-699 (2008).

44 Arash A. Mostofi, Jonathan R. Yates, Giovanni Pizzi, Young-Su Lee, Ivo Souza, David Vanderbilt, and Nicola Marzari, "An updated version of wannier90: A tool for obtaining maximally-localised wannier functions," Computer Physics Communications 185, 2309-2310 (2014)

45 V.M. Edelstein, "Spin polarization of conduction electrons induced by electric current in two-dimensional asymmetric electron systems," Solid State Communications 73, 233 - 235 (1990)

40 L. L. Tao and Evgeny Y. Tsymbal, "Persistent spin texture enforced by symmetry," Nature Communications 9, 2763 (2018).

47 Stefanos Kourtis, Jian Li, Zhijun Wang, Ali Yazdani, and B. Andrei Bernevig, "Universal signatures of Fermi arcs in quasiparticle interference on the surface of Weyl semimetals," Phys. Rev. B 93, 041109 (2016). 